FORMATION Formation emploi

Revue française de sciences sociales

125 | Janvier-Mars 2014

Pêle-Mêle

\title{
Les professions intermédiaires : ni cadres, ni ouvriers, ni employés
}

Présentation de l'ouvrage Les professions intermédiaires. Des métiers d'interface au cœur de l'entreprise

\section{Julien Damon}

\section{(2) OpenEdition}

Journals

Édition électronique

URL : http://journals.openedition.org/formationemploi/4163

DOI : 10.4000/formationemploi.4163

ISSN : 2107-0946

Éditeur

La Documentation française

Édition imprimée

Date de publication : 25 mars 2014

ISSN : 0759-6340

Référence électronique

Julien Damon, «Les professions intermédiaires : ni cadres, ni ouvriers, ni employés », Formation emploi [En ligne], 125 | Janvier-Mars 2014, mis en ligne le 10 avril 2014, consulté le 10 décembre 2020. URL : http://journals.openedition.org/formationemploi/4163; DOI : https://doi.org/10.4000/ formationemploi.4163 


\section{Note de lecture}

\section{Les professions intermédiaires: ni cadres, ni ouvriers, ni employés}

par Julien Damon

Professeur associé à sciences po

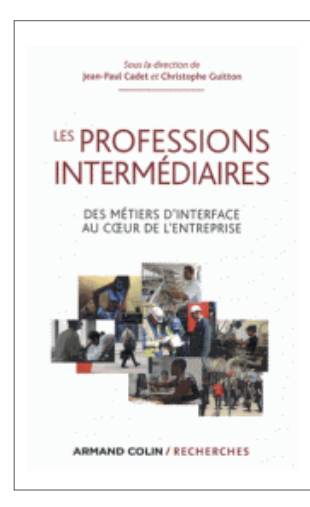

Analyser les classes moyennes relève d'une logique " ni ni ». Ces catégories comprennent un pluriel qui désigne leur hétérogénéité. Elles rassemblent des personnes et ménages qui ne sont, en termes de revenu, ni riches, ni pauvres. Et qui vivent, en termes de localisation, dans des quartiers qui ne sont ni huppés, ni défavorisés. Assez classiquement, les travaux français sur ce sujet passent aussi par les professions et catégories socioprofessionnelles (PCS). En l'espèce, les classes moyennes regroupent des actifs qui ne sont ni exécutants, ni dirigeants, avec des statuts souvent ambivalents par rapport à celui de cadre.

Une trentaine d'auteurs, la plupart enseignants-chercheurs, emmenés par Jean-Paul Cadet et Christophe Guitton, se sont penchés sur ces catégories professionnelles " ni ni ", connues, par la statistique publique, comme les professions intermédiaires. Les classes moyennes, en général, étant souvent décrites comme se situant au cœur de la société, les professions intermédiaires sont comprises comme au cœur de l'entreprise.

Mobilisant les moyens et le réseau de recherche du Céreq (Centre d'études et de recherches sur les qualifications), une trentaine de contributions traite des questions d'emploi, de travail et de formation. Elles s'appuient sur un ambitieux projet collectif de recherche qui met autant au jour le halo de la catégorie que la diversité des activités et réalités de métiers que l'on trouve dans tous les secteurs (construction automobile, santé, informatique ou banque). Qui sont, que sont et que font ces professions intermédiaires?

Ces pages constituent le panorama le plus actuel de ce que l'INSEE, avant la révision de sa nomenclature des PCS en 1982, baptisait les « cadres moyens ». Le qualificatif, qui pouvait être jugé dépréciatif, les positionnait d'emblée au sein des classes moyennes. C'est toujours la place qu'elles occupent sur le prisme des positions sociales. Toujours apprécié dans un " ni ni ", entre exécution et conception, cet archipel ou cette galaxie (pour reprendre 
deux des expressions employées par les auteurs) de métiers a donné lieu, pendant une demie-décennie, à une "Enquête sur les professions intermédiaires en entreprises " (EPIE) qui a porté sur 65 métiers différents dans une trentaine d'entreprises. Qu'il s'agisse d'agents de maîtrise, de techniciens, de commerciaux, de personnels administratifs et infirmiers, ces professionnels se trouvent assurément au cœur de l'entreprise, mais aussi au cœur des thématiques des ressources humaines, en problématique comme en volume.

Suivant la logique du programme EPIE, le livre s'organise selon un triptyque. Il a été conçu et se lit comme un triple état des savoirs, des lieux et des problématiques. Plongée dans des univers professionnels aux contenus et carrières qui diffèrent largement (dans un contexte de "brouillard statutaire ", pour reprendre l'expression de Christine Guégnard), cette investigation trouve son unité dans la fonction d'interface (de charnière, disent aussi certains des auteurs) qui caractérise ces professions. Celles-ci peuvent relever, en ce qui concerne les fonctions assurées, du management, de la technique, de l'administration ou du commercial. Ces différentes fonctions ayant d'ailleurs, comme l'observe Christophe Guitton, tendance à s'intégrer dans une polyvalence difficile autant à caractériser qu'à faire efficacement vivre.

L'identité est peu assurée pour le quart de la population salariée qui est ainsi recensé. Et pourtant, ce segment du salariat a vu grossir ses effectifs de moitié entre 1985 et 2010. C'est dire l'importance de s'y pencher avec détermination. Plus féminisé, avec un vieillissement moins marqué, un accès privilégié à la formation continue et toujours des capacités de promotion, le groupe demeure avant tout un agrégat statistique rassemblant des fonctions, des diplômes et des positions disparates. On a beau chercher à partir de tous les critères possibles - diplôme, rang dans l'entreprise, type d'activité - il n'est pas possible d'agréger la population des intermédiaires (disons cela ainsi) dans des frontières parfaitement définies. Toujours se pose la question des lisières, plus ou moins perméables avec les catégories des cadres, des employés et des ouvriers. D’infinies difficultés se posent pour le codage dans des groupes socioprofessionnels, en particulier dans le secteur privé, qui ne dispose pas d'une catégorie B (entre A et C donc) comme dans la fonction publique.

L'ouvrage fait utilement le point sur les débats théoriques et empiriques, notamment quant à savoir s'il faut véritablement parler de professions, ou bien s'il faut se cantonner à observer des positions. Dans la plupart des domaines d'activité, des professions " canoniques " incarnent ce qui est intermédiaire, mais dans tous les cas, des périphéries (plus ou moins larges) coexistent, au-dessus ou en dessous (c'est selon). Il n'en va pas seulement de controverses savantes et de chicaneries statistiques. Le propos est bien de savoir comment décrire la réalité, mais aussi de pouvoir faire vivre intelligemment et justement des conventions collectives. Charles Gadea souligne ici l'importance du modèle de la profession pour bien se situer et résister à une dilution 
dans le "managérialisme» (l'extension des pratiques et objectifs du management dans toutes les sphères d'activité).

Au-delà des débats et des clarifications (qui intéressent autant la recherche que la pratique), l'ouvrage étudie en profondeur certaines professions intermédiaires. Ces contributions sont de petites monographies, bien renseignées et généralement rapportées aux enseignements et observations plus générales à tirer de l'enquête EPIE. Il en va ainsi du portrait de responsables d'unité de montage ou d'usinage dans un groupe automobile. Du décorticage de la fiche de poste à l'analyse des objectifs, en passant par la description des activités quotidiennes et des outils de gestion (parfois trop lourds), ce type de description met en lumière le quotidien et les tensions de tels métiers, et, surtout, de telles positions.

Des interviews nourrissent quelques-unes des parties. Elles fournissent des informations déterminantes, par exemple sur les raisons qui peuvent pousser des infirmières à ne pas vouloir devenir cadres (refus de s'éloigner des patients, mais aussi usure généralisée et choix d'une toute autre voie).

Dans une entreprise de restauration rapide, le manager à la tête de l'unité est en pleine responsabilité de son équipe et de son fast-food, mais il est contraint par des procédures standardisées. Le fait d'être intermédiaire dans ce contexte est à comprendre à la fois comme spécificité des positions occupées, mais aussi comme étape dans une carrière (très méritocratique) permettant de progresser dans l'intermédiaire, et, pour quelques-uns, vers la catégorie de cadre. Anne Gillet, quant à elle, traite du responsable d'équipe de ligne. Avançant une autre forme de "ni ni ", elle écrit que les agents de maîtrise se trouvent entre "le marteau et l'enclume ». Le manager de proximité doit concilier commandement et proximité avec des équipes qui, par construction, sont dispersées et mobiles. Sophie Divay s'intéresse aux infirmières qui présentent des parcours très contrastés, avec notamment de possibles bascules entre le public et le privé. Encore dans une forme de «ni ni ", certaines professions intermédiaires se situent entre le conseil et la vente aux clientèles. C'est le cas du commercial dans le secteur automobile, comme du chargé de clientèle dans la banque. Dans les métiers qui relèvent davantage de la fonction « administration " (dont le livre signale l'inflation), on repère une polyvalence de tâches et un renouveau des compétences que masque certainement une désignation obsolète. Il s'ensuit, comme l'estime Anne Moysan-Louazel, une " professionnalisation problématique».

Au total, la lecture de ce vaste tour d'horizon confirme bien l'impression de flou - car avec des frontières très mouvantes - de l'ensemble désigné comme professions intermédiaires. Avec une posture généralement critique sur les conséquences de la diffusion du New Public Management et des modes managériales, cette très utile collection organisée de textes montre clairement les difficultés et interrogations de ces professionnels, de ceux qui les observent et de ceux qui les gèrent. Ces 400 pages doivent incontestablement être positionnées comme la source autorisée sur ces professions intermédiaires, sans cacher 
la quasi-impossibilité de dégager définitivement leur unité. José Rose conclue d'ailleurs que "le sentiment de la diversité voire de l'hétérogénéité de la catégorie l'emporte".

Ce volume est incontournable pour qui porte de l'intérêt aux professions intermédiaires. Il s'insère dans une bibliothèque sur les étagères des transformations du monde du travail, que l'ensemble de ces analyses vient documenter à partir d'un prisme particulier, mais central, dans le monde de l'entreprise.

\section{Référence de l'ouvrage}

Cadet J.-P., Guitton C. (dir.) (2013), Les professions intermédiaires. Des métiers d'interface au coeur de l'entreprise, Paris, Armand Colin, coll. "Recherches », 394 p. 